\title{
CONTRIBUIÇÃO A APLICAÇÃO DAS WAVELETS NA ELETROSTÁTICA
}

\author{
A. A. Belardi ${ }^{1}$ \\ belardi@fei.edu.br
}

\author{
J. R. Cardoso ${ }^{2}$ \\ cardoso@pea.usp.br
}

\author{
C. A. F. Sartori ${ }^{2}$ \\ sartori@pea.usp.br
}

\author{
${ }^{1}$ Centro Universitário da FEI \\ Av. Humberto de Alencar Castelo Branco, n 3972, 09850-901, São Bernardo do Campo, SP, Brasil \\ ${ }^{2}$ Escola Politécnica da Universidade de São Paulo \\ Av. Prof. Luciano Gualberto, $n^{\circ}$ 380, 05508-900, Trav. 3, São Paulo, Brasil
}

\begin{abstract}
This work presents the methodology from the determination the charge's superficial density, in two simple structure a to straight thread and in plane plates, both finite and submitted to a constant potential. That involves the method of the moments using as expansion function the wavelets instead of the pulse function, in order to reach a good precision and reducing the computational execution time.
\end{abstract}

We also intends to take advantages of the wavelets application through the Cholesky decomposition, talking about formation of scattered matrixes, and the detection of nulls values.

KEYWORDS: Wavelets.

\section{RESUMO}

Este trabalho apresenta uma metodologia para a determinação da densidade superficial de carga em duas estruturas simples, um fio reto finito e uma chapa plana quadrada ambas submetidas a um potencial constante. A metodologia envolve o método dos momentos, utilizando como função de expansão as wavelets de Haar ao invés da

Artigo Submetido em 19/12/03

1a. Revisão em 13/04/04;

2a. Revisão em 31/05/04;

Aceito sob recomendação do Editor Associado

Prof. Dr. Jose Antenor Pomilio função pulso, visando a obtenção de uma boa precisão e de uma redução no esforço computacional. Através de um artifício matemático, podemos selecionar um limiar em porcentagem que permite descartar valores próximos de zero, sem grandes variações no resultado final. Apresentamos também as vantagens computacionais, que podemos obter através da decomposição de Cholesky.

PALAVRAS-CHAVE: momentos, wavelets, eletrostática

\section{INTRODUÇÃO}

\subsection{Método dos Momentos}

Método dos Momentos, (Harrington,1968), é um método generalizado baseado no princípio dos resíduos ponderados. Este método engloba muitos métodos específicos conhecidos podendo-se mencionar o método da simulação de carga, o método dos elementos finitos, que são considerados como um dos casos especiais do método dos momentos. Qualquer método em que os coeficientes de uma equação podem ser determinados através de um sistema matricial, pode ser interpretado como uma variação do método dos momentos.

Assim, os passos para a aplicação do método dos momentos podem ser sistematizados da seguinte forma: 
a) Selecionar uma função aproximada para substituir a função desconhecida;

b) Selecionar uma função de expansão e de ponderação;

c) Realizar o produto interno entre a função de expansão e de ponderação para obtermos a matriz resultante;

d) Solução da equação matricial através de um programa computacional, para obtermos a solução aproximada.

A base do método dos momentos é aproximar uma função do tipo :

$$
\mathrm{f}(\mathrm{x})=\sum_{\mathrm{n}} \alpha_{\mathrm{n}} \boldsymbol{L} \mathrm{g}_{\mathrm{n}}
$$

sendo que $\alpha_{n}$ são constantes não conhecidas, $g_{n}$ é uma função de expansão e $\boldsymbol{L}$ é um operador matemático. Alguns fatores adicionais como a precisão na solução, a facilidade da solução da matriz resultante, o tamanho da matriz etc., dependem diretamente da escolha da função base e da função de ponderação. Quando adotamos a função de ponderação igual à função base, o resultado obtido na aplicação do método dos momentos é chamado de método de Galerkin. Fazendo-se o produto interno com uma função de ponderação e utilizando-se a notação matricial, temos que $[\mathrm{A}]^{*}[\alpha]=[\mathrm{B}]$, sendo $[\alpha]$ um vetor coluna composto pelos coeficientes desconhecidos da solução aproximada.

\subsection{Wavelets}

Segundo Morettin e Rabello,(1999) a análise das wavelets, já era utilizada por vários cientistas como uma alternativa à análise de Fourier clássica. As wavelets de J. S. Liénard e de $\mathrm{X}$. Rodet estão relacionadas ao tratamento numérico de sinais acústicos e as de J. Morlet foram desenvolvidas para estocar e interpretar os sinais sísmicos.

Nos últimos dez anos, o interesse pelas wavelets cresceu assustadoramente a partir de 1985 , quando foi dado um novo impulso a esta teoria através da contribuição de matemáticos e especialistas em processamento de sinal.

De uma maneira geral, a wavelet pode ser definida por :

$\psi_{\mathrm{a}, \mathrm{b}}(\mathrm{x})=|\mathrm{a}|^{-1 / 2} \psi\left(\frac{\mathrm{x}-\mathrm{b}}{\mathrm{a}}\right) \quad \mathrm{a}, \mathrm{b} \in \mathrm{R}, \mathrm{a} \neq 0$
A função $\Psi$ é chamada wavelet mãe, sendo os valores de a e $\mathrm{b}$ definidos por $\mathrm{a}=2^{-\mathrm{j}}$ e $\mathrm{b}=\mathrm{k} 2^{-\mathrm{j}}$ com $\mathrm{j}$ e $\mathrm{k}$ pertencentes ao conjunto dos números inteiros relativos.

\subsubsection{Wavelets Unidimensionais}

Considerando-se o espaço $\mathrm{L}^{2}(\mathrm{R})$, de todas funções mensuráveis de quadrado integrável sobre $R$, e considerando-se uma base ortogonal gerada por $\Psi$, para qualquer $\mathrm{f}(\mathrm{x})$ de quadrado integrável sobre $\mathrm{R}$ teremos :

$$
f(x)=\sum_{j=-\infty}^{\infty} \sum_{k=-\infty}^{\infty} c_{j, k} \psi_{j, k}(x)
$$

Um exemplo de wavelet, é a função de Haar que é definida conforme Fig. 1:

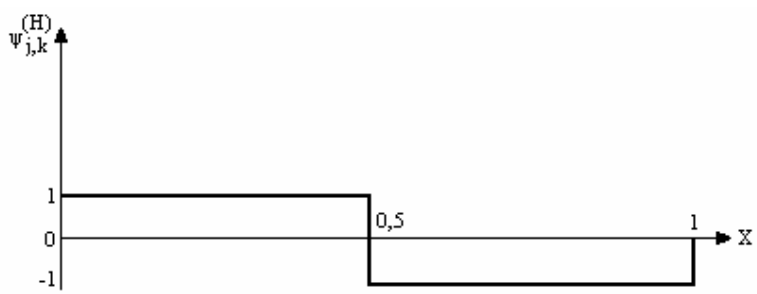

Fig. 1 - Função de Haar definida no intervalo de 01

A wavelet de Haar é definida por duas famílias de wavelet, a mãe e a pai, sendo esta última representada por $\phi$ e referenciada na literatura (Aboufadel e Schlicker 1999) como função escala e definida por:

$$
\phi^{(\mathrm{H})}(\mathrm{x})= \begin{cases}1 & 0 \leq \mathrm{x}<1 \\ 0 & \text { caso contrário }\end{cases}
$$

A Fig. 2, mostra graficamente os intervalos e as amplitudes para wavelet de Haar unidimensional, até o nível dois de resolução, representando a amplitude da função obtida através da variação do nível $\mathrm{j}$ e do deslocamento $\mathrm{k}$.

Considerando-se as wavelets mãe (2) e pai (4), a função $\mathrm{f}(\mathrm{x})$ pode ser escrita por :

$\mathrm{f}(\mathrm{x})=\sum_{\mathrm{k}=-\infty}^{\infty} \mathrm{c}_{0, \mathrm{k}} \phi^{(\mathrm{H})}(\mathrm{x})+\sum_{\mathrm{j}=-\infty}^{\infty} \sum_{\mathrm{k}=-\infty}^{\infty} \mathrm{c}_{\mathrm{j}, \mathrm{k}} \psi_{\mathrm{j}, \mathrm{k}}^{(\mathrm{H})}(\mathrm{x})$ 


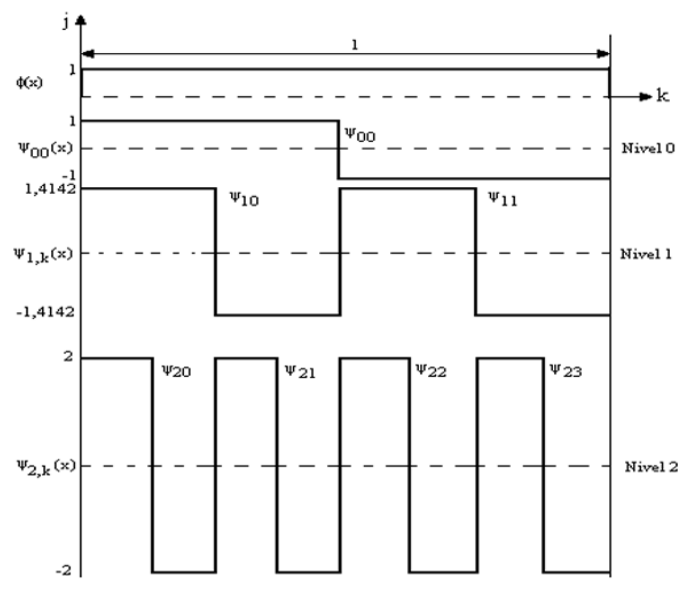

Fig. 2 - Comportamento da função de Haar unidimensional até nível dois de resolução

\subsubsection{Wavelets Bidimensionais}

Podemos construir bases de wavelets bidimensionais, (Morettin,1999 e Chui,1992) através de uma base em duas dimensões com uma única escala, ou também através de duas bases de uma dimensão, com escalas distintas para cada dimensão. Uma outra possibilidade (Newland,1993) é de se trabalhar com a combinação dos produtos entre cada um dos dois eixos, com escalas distintas para cada dimensão. Assim para um ponto $\mathrm{P}$ qualquer, até o nível 1 de resolução teremos:

$\psi^{(\mathrm{H})}(\mathrm{x})=[\phi(\mathrm{x}) \psi(\mathrm{x}) \psi(2 \mathrm{x}) \psi(2 \mathrm{x}-1)]$

$\psi^{(\mathrm{H})}(\mathrm{y})=[\phi(\mathrm{y}) \psi(\mathrm{y}) \psi(2 \mathrm{y}) \psi(2 \mathrm{y}-1)]$

A Fig. 3 mostra a decomposição da amplitude e da escala, até o nível 1 de resolução, da wavelet de Haar para duas dimensões.

Para cada um dos eixos, $\mathrm{x}$ é dado por $\psi^{(\mathrm{H})}(\mathrm{x})$ e y dado por $\psi^{(\mathrm{H})}(\mathrm{y})$, resultando nas seguintes contribuições :

$\psi^{(\mathrm{H})}(\mathrm{x})=\left[\phi(\mathrm{x}) \psi_{00}(\mathrm{x}) \psi_{10}(\mathrm{x})\right]$

$\psi^{(\mathrm{H})}(\mathrm{y})=\left[\phi(\mathrm{y}) \psi_{00}(\mathrm{y}) \psi_{10}(\mathrm{y})\right]$

O conjunto de coeficientes das wavelets obtidos juntamente com a contribuição de cada uma delas, será utilizado para determinar a função $\mathrm{f}(\mathrm{x}, \mathrm{y})$ em cada ponto.

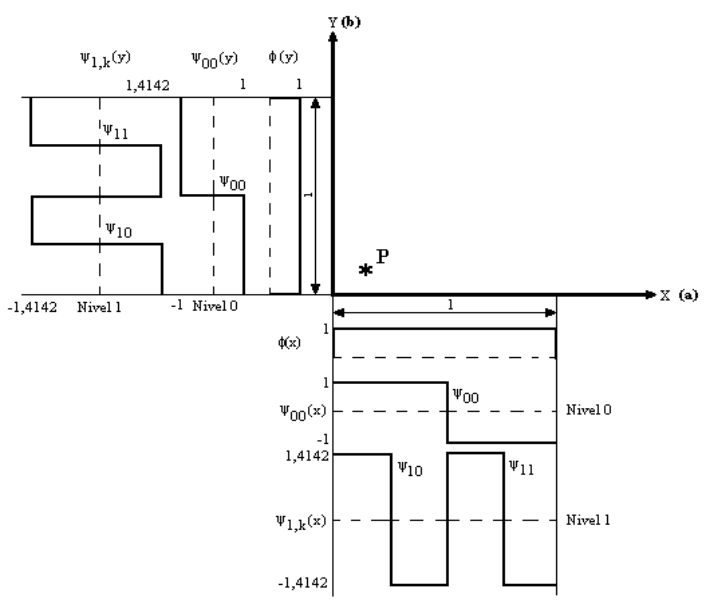

Fig. 3 Comportamento da função de Haar bidimensional até o nível um de resolução

\section{DESENVOLVIMENTO MATEMÁTICO}

A seguir, apresentamos o desenvolvimento matemático para a aplicação do método dos momentos na determinação da densidade superficial de carga para um fio reto finito (caso unidimensional) e para uma chapa quadrada (caso bidimensional), ambos submetidos a um potencial constante.

\subsection{Caso Unidimensional}

Primeiramente, realizamos um estudo da distribuição de cargas elétricas em um fio reto finito, com diâmetro desprezível, resultando em uma equação de uma variável do tipo (Constantine, 1989) :

$\mathrm{V}(\mathrm{r})=\frac{1}{4 \pi \varepsilon} \int \frac{\rho\left(\mathrm{r}^{\prime}\right)}{\mathrm{R}\left(\mathrm{r}, \mathrm{r}^{\prime}\right)} \mathrm{dl}$

Como o fio é reto e finito e posicionado no eixo $\mathrm{x}$, as observações em relação aos eixos y e z são consideradas iguais a zero. $\mathrm{O}$ potencial eletrostático é escrito por :

$\mathrm{V}(\mathrm{x}, \mathrm{ye} \mathrm{z}=0)=\mathrm{V}(\mathrm{r})=\frac{1}{4 \pi \varepsilon} \int \frac{\rho\left(\mathrm{r}^{\prime}\right)}{\mathrm{R}\left(\mathrm{x}, \mathrm{x}^{\prime}\right)} \mathrm{dl}^{\prime}$

Para o cálculo do potencial e de outras grandezas físicas tais como capacitância, campo elétrico, etc., devemos calcular a densidade linear de carga, que é uma grandeza desconhecida, a qual está dentro de um operador matemático.

A determinação da densidade linear de carga, pode ser aproximada através da expansão em $\mathrm{N}$ termos, compostos pela somatória dos coeficientes multiplicados pela função 
de expansão e de ponderação. Dividindo-se o fio em N segmentos iguais, teremos que o comprimento de cada parte valerá $\Delta=\mathrm{L} / \mathrm{N}$. Aplicando-se a função de ponderação como sendo $\mathrm{W}_{\mathrm{m}}=\delta\left(\mathrm{x}-\mathrm{x}_{\mathrm{m}}\right)=1$, o produto interno no ponto será dado por:

$\mathrm{V}(\mathrm{r})_{\text {Ponto }}=\delta\left(\mathrm{x}-\mathrm{x}_{\mathrm{m}}\right) \frac{1}{4 \pi \varepsilon} \sum_{\mathrm{n}-1}^{\mathrm{N}} \alpha_{\mathrm{n}} \int_{0}^{\mathrm{L}} \frac{\mathrm{g}_{\mathrm{n}}\left(\mathrm{x}^{\prime}\right)}{\sqrt{\left(\mathrm{x}-\mathrm{x}^{\prime}\right)^{2}+\mathrm{a}^{2}}} \mathrm{dx}^{\prime}$

Admitindo-se que a carga obtida em cada subdivisão do fio está posicionada no centro de gravidade de cada divisão, substituindo-se o valor de $\mathrm{x}$ pela distância da carga considerada em relação à observada, teremos uma integral que é somente função de x'. Utilizando a notação matricial, cada $\mathrm{Z}_{\mathrm{mn}}$ será definido por :

$$
\mathrm{Z}_{\mathrm{mn}}=\int_{0}^{\mathrm{L}} \frac{\mathrm{g}_{\mathrm{n}}\left(\mathrm{x}^{\prime}\right)}{\sqrt{\left(\mathrm{x}_{\mathrm{m}}-\mathrm{x}^{\prime}\right)^{2}+\mathrm{a}^{2}}} \mathrm{dx}
$$

\subsection{Caso Bidimensional}

Através da metodologia apresentada anteriormente, o potencial em uma chapa plana finita com espessura desprezível gera um potencial dado por :

$V(x, y)=\frac{1}{4 \pi \varepsilon} \int_{-a}^{a} d x^{\prime} \int_{-b}^{b} \frac{\rho\left(x^{\prime}, y^{\prime}\right)}{\sqrt{\left(x-x^{\prime}\right)^{2}+\left(y-y^{\prime}\right)^{2}}} d y^{\prime}$

Dividindo-se cada um dos eixos em segmentos iguais e admitindo que a distribuição de carga esteja concentrada no centro de gravidade de cada área $\Delta \mathrm{S}_{\mathrm{n}}$, a influência entre a carga localizada na posição $(\mathrm{x}, \mathrm{y})$, em relação a outra determinada pelas coordenadas (x',y'), é definida pela distância $\mathrm{R}$ entre as cargas. Aplicando-se o método dos momentos, a distribuição de carga pode ser aproximada pela seguinte equação :

$$
\begin{aligned}
\mathrm{V}(\mathrm{x}, \mathrm{y})_{\text {Ponto }}= & \left\langle\mathrm{W}_{\mathrm{m}}, \mathrm{f}, \operatorname{Lg}\right\rangle=\delta\left(\mathrm{x}-\mathrm{x}_{\mathrm{m}}\right) \delta\left(\mathrm{y}-\mathrm{y}_{\mathrm{m}}\right) \times \\
& \frac{1}{4 \pi \varepsilon} \int_{-\mathrm{a}}^{\mathrm{a}} \mathrm{dx} \mathrm{x}^{\prime} \int_{-\mathrm{b}}^{\mathrm{b}} \frac{\sum_{\mathrm{n}=1}^{\mathrm{N}} \alpha_{\mathrm{n}} \mathrm{g}_{\mathrm{n}}\left(\mathrm{x}^{\prime}, \mathrm{y}^{\prime}\right)}{\sqrt{\left(\mathrm{x}-\mathrm{x}^{\prime}\right)^{2}+\left(\mathrm{y}-\mathrm{y}^{\prime}\right)^{2}}} d y^{\prime}
\end{aligned}
$$

Substituindo-se o valor de x e y pela distância $\mathrm{R}$, para cada ponto $\mathrm{x}_{\mathrm{m}}$ e $\mathrm{y}_{\mathrm{m}}$, teremos uma integral que é somente função de $x^{\prime}$ e $y^{\prime}$.

$$
\mathrm{Z}_{\mathrm{mn}}=\int_{-\mathrm{a}}^{\mathrm{a}} \mathrm{dx} \mathrm{x}^{\prime} \int_{-\mathrm{b}}^{\mathrm{b}} \frac{\mathrm{g}_{\mathrm{n}}\left(\mathrm{x}^{\prime}, \mathrm{y}^{\prime}\right)}{4 \pi \varepsilon \sqrt{\left(\mathrm{x}_{\mathrm{m}}-\mathrm{x}^{\prime}\right)^{2}+\left(\mathrm{y}_{\mathrm{m}}-\mathrm{y}^{\prime}\right)^{2}}} \mathrm{dy} \mathrm{y}^{\prime}
$$

\section{APLICAÇÕES}

\subsection{Aplicação Unidimensional - Fio reto finito}

Para verificarmos o comportamento das wavelets no método dos momentos, realizamos um estudo em um fio reto finito. Neste caso, utiliza-se como função de expansão a wavelet de Haar e como função de ponderação, a função Delta de Dirac.

O potencial em um fio reto finito, poderá ser escrito por :

$\mathrm{V}(\mathrm{r})=\frac{1}{4 \pi \varepsilon}\left[\int_{0}^{\mathrm{L}} \frac{\mathrm{c}_{0} \phi(\mathrm{x})}{\mathrm{R}\left(\mathrm{x}, \mathrm{x}^{\prime}\right)} \mathrm{dx}+\int_{0}^{\mathrm{L}} \frac{\sum_{\mathrm{n}=1}^{\mathrm{N}} \mathrm{c}_{\mathrm{j}, \mathrm{k}} \psi_{\mathrm{j}, \mathrm{k}}^{(\mathrm{H})}\left(\mathrm{x}^{\prime}\right)}{\mathrm{R}\left(\mathrm{x}, \mathrm{x}^{\prime}\right)} \mathrm{dx}\right]$

Dividimos o fio com comprimento de $1 \mathrm{~m}$ e diâmetro de 0,0001m, em oito segmentos iguais de acordo com a Fig. 4. Para a carga situada na primeira subdivisão, consideramos, as contribuições $\phi(\mathrm{x}), \psi_{00}(\mathrm{x}), \psi_{10}(\mathrm{x})$ e $\psi_{20}(\mathrm{x})$.

Para o primeiro ponto, considerando-se todas as subdivisões, teremos :

$$
\begin{aligned}
\text { Ponto }_{1} & =\mathrm{c}_{0} \int_{0}^{\mathrm{L}} \frac{1}{\sqrt{\left(\mathrm{x}_{\mathrm{m}}-\mathrm{x}^{\prime}\right)^{2}+\mathrm{a}^{2}}} \mathrm{dx}+\mathrm{c}_{00} \int_{0}^{\Delta} \frac{1}{\sqrt{\left(\mathrm{x}_{\mathrm{m}}-\mathrm{x}^{\prime}\right)^{2}+\mathrm{a}^{2}}} \mathrm{dx}+ \\
& +\mathrm{c}_{10} \int_{0}^{\Delta} \frac{\sqrt{2}}{\sqrt{\left(\mathrm{x}_{\mathrm{m}}-\mathrm{x}^{\prime}\right)^{2}+\mathrm{a}^{2}}} \mathrm{dx}+\mathrm{c}_{20} \int_{0}^{\Delta} \frac{2}{\sqrt{\left(\mathrm{x}_{\mathrm{m}}-\mathrm{x}^{\prime}\right)^{2}+\mathrm{a}^{2}}} \mathrm{dx}
\end{aligned}
$$

Resolvendo-se a integral e considerando-se $\mathrm{x}_{\mathrm{m}}$ com sendo a distância da carga origem em relação à fonte, teremos que cada $Z_{\mathrm{mn}}$ será definido por :

$\mathrm{Z}_{\mathrm{mn}}=\ln \frac{\mathrm{n} \Delta-\mathrm{x}_{\mathrm{m}}+\sqrt{\left(\mathrm{x}_{\mathrm{m}}^{2}-2 \mathrm{x}_{\mathrm{m}} \mathrm{n} \Delta+\mathrm{a}^{2}+\mathrm{n}^{2} \Delta^{2}\right)}}{\mathrm{n} \Delta-\Delta-\mathrm{x}_{\mathrm{m}}+\sqrt{\left(\mathrm{x}_{\mathrm{m}}^{2}-2 \mathrm{x}_{\mathrm{m}} \mathrm{n} \Delta+2 \mathrm{x}_{\mathrm{m}} \Delta+\mathrm{a}^{2}+\mathrm{n}^{2} \Delta^{2}-2 \Delta^{2} \mathrm{n}+\Delta^{2}\right)}}$ 


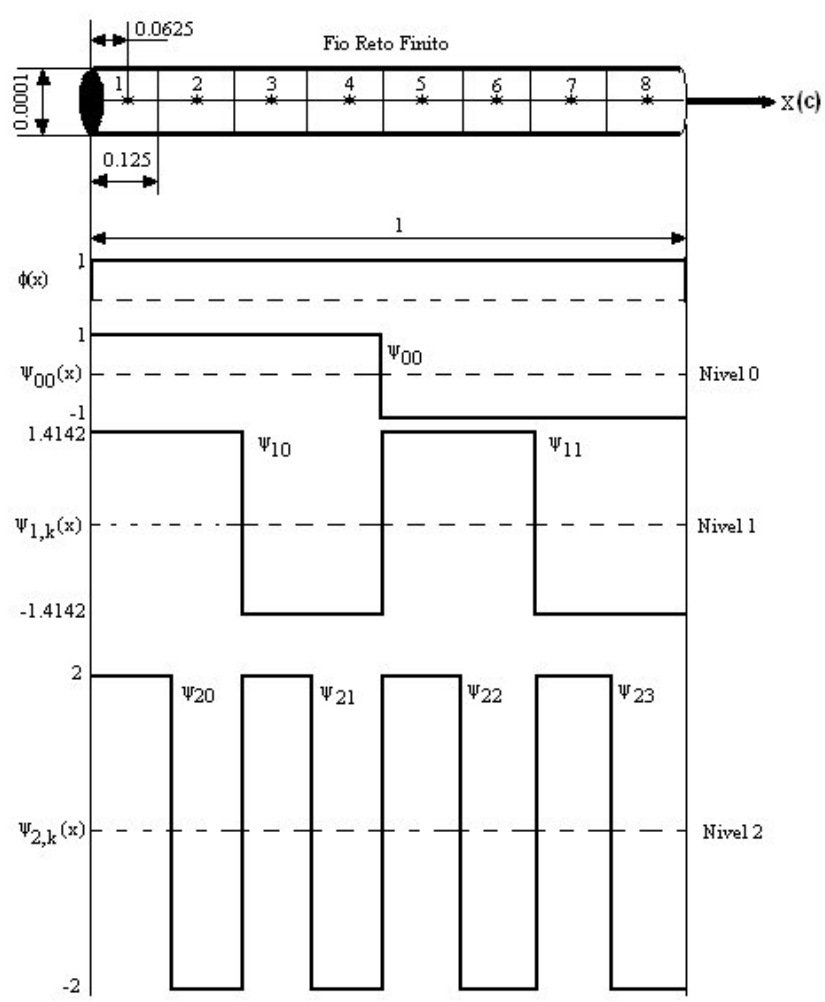

Fig. 4 - Contribuição de cada um dos níveis em relação ao número de subdivisões

O cálculo da densidade linear de carga em cada ponto, utilizando-se como função de expansão a wavelet de Haar, será dado por :

$\rho(\mathrm{x})_{\text {Ponto P }}=\mathrm{c}_{0} \phi(\mathrm{x})+\mathrm{c}_{00} \psi_{00}(\mathrm{x})+\mathrm{c}_{10} \psi_{10}(\mathrm{x})+\ldots+\mathrm{c}_{\mathrm{jk}} \psi_{\mathrm{jk}}(\mathrm{x})$

A Tabela 1 apresenta os resultados obtidos da densidade linear de carga para um fio reto com comprimento de $1,0 \mathrm{~m}$, dividido em 16 segmentos iguais, em função do nível de resolução ( $\mathrm{j}$ ) e do deslocamento $(\mathrm{k})$.

\subsection{Aplicação Bidimensional - Chapa Plana}

O tratamento da wavelet de Haar bidimensional é dado pela combinação dos produtos da função no ponto em relação a cada eixo, considerando-se os seus respectivos coeficientes.

O potencial de uma chapa plana finita poderá ser escrito por:

$$
\begin{aligned}
& V(x, y) 4 \pi \varepsilon=a_{j} b_{j} \int_{-a}^{a} \int_{-b}^{b} \frac{\phi(x, y)}{\sqrt{\left(x-x^{\prime}\right)^{2}+\left(y-y^{\prime}\right)^{2}}}+ \\
& +\left[\sum_{j=-\infty}^{\infty} \sum_{k=-\infty}^{\infty} a_{j, k} b_{j, k} \int_{-a}^{a} \int_{-b}^{b} \frac{\psi_{j, k}^{(H)}(x, y)}{\sqrt{\left(x-x^{\prime}\right)^{2}+\left(y-y^{\prime}\right)^{2}}} d x d y\right]
\end{aligned}
$$

\section{TABELA 1 - VALOR DA DENSIDADE LINEAR DE CARGA (pC/m) DE UM FIO RETO FINITO EM FUNÇÃO DOS NÍVEIS DE RESOLUÇÃO}

\begin{tabular}{|c|c|c|c|c|}
\hline \multirow[t]{2}{*}{ Ponto } & \multicolumn{3}{|c|}{ wavelet de Haar (Nível) } & \multirow[t]{2}{*}{ Pulso } \\
\hline & 2 & 3 & 4 & \\
\hline 1 & 8,835 & 9,376 & 9,957 & 9,957 \\
\hline 2 & 8,835 & 9,376 & 8,764 & 8,764 \\
\hline 3 & 8,835 & 8,274 & 8,411 & 8,411 \\
\hline \multirow[t]{2}{*}{4} & 8,835 & 8,274 & 8,219 & 8,219 \\
\hline & 7,970 & 8,059 & 8,102 & 8,102 \\
\hline$\ldots$ & $\ldots$ & $\ldots$ & $\ldots$ & $\ldots$ \\
\hline 12 & 7,970 & 8,059 & 8,102 & 8,102 \\
\hline 13 & 8,835 & 8,274 & 8,219 & 8,219 \\
\hline 14 & 8,835 & 8,274 & 8,411 & 8,411 \\
\hline 15 & 8,835 & 9,376 & 8,764 & 8,764 \\
\hline 16 & 8,835 & 9,376 & 9,957 & 9,957 \\
\hline
\end{tabular}

Função de Expansão

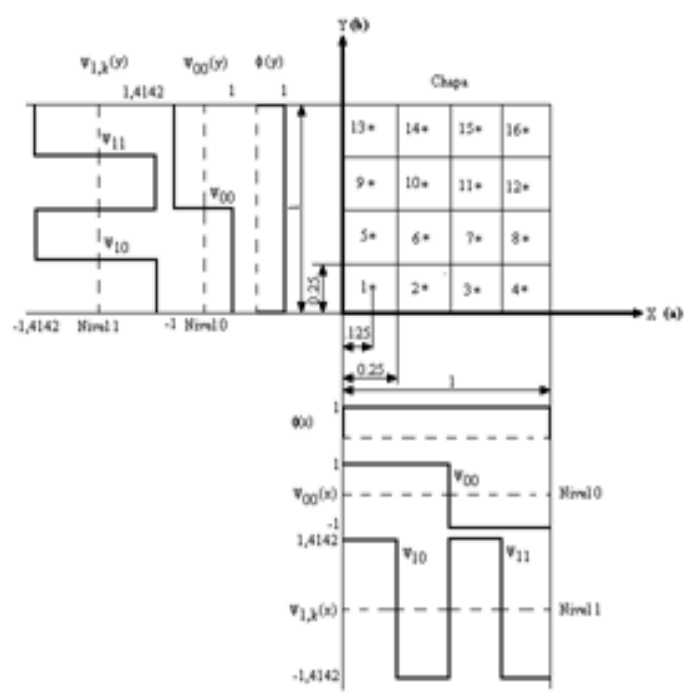

Fig. 5 - Contribuição da wavelet em relação a cada ponto formado pela subdivisão 
A placa foi dividida em passos de 0,25 , tanto no eixo $\mathrm{x}$ como no $\mathrm{y}$. A Fig. 5 mostra estas divisões considerando-se o nível um de resolução.

Para a carga situada na primeira subdivisão, serão consideradas as contribuições das seguintes wavelets de Haar até o nível 2 de resolução :

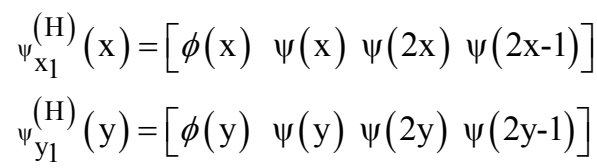

Fazendo-se a função de ponderação igual a um e admitindose que a densidade de carga em uma pequena área $\Delta \mathrm{S}_{\mathrm{n}}$ é constante, conhecendo-se o valor do potencial podemos determinar a solução aproximada, ou seja:

$\mathrm{m}=\mathrm{n} \Rightarrow \mathrm{Z}_{\mathrm{mn}}=\frac{2 \mathrm{~b}}{\pi \varepsilon} \ln (1+\sqrt{2}), \mathrm{b}=$ lado da subdivisão

$\mathrm{m} \neq \mathrm{n} \Rightarrow \mathrm{Z}_{\mathrm{mn}}=\frac{\Delta \mathrm{S}_{\mathrm{n}}}{4 \pi \varepsilon \sqrt{\left(\mathrm{x}_{\mathrm{m}}-\mathrm{x}_{\mathrm{n}}\right)^{2}+\left(\mathrm{y}_{\mathrm{m}}-\mathrm{y}_{\mathrm{n}}\right)}}$

A contribuição de cada wavelet no ponto considerado será dada por:

$$
\begin{aligned}
& \operatorname{EXP}(\mathrm{P} 1)=c_{0}+c_{1}+\sqrt{2} c_{2}+c_{4}+c_{5}+\sqrt{2} c_{6}+\sqrt{2} c_{8}+\sqrt{2} c_{9}+\sqrt{2} \sqrt{2} c_{10} \\
& \operatorname{EXP}(P 2)=c_{0}+c_{1}+\sqrt{2} c_{2}+c_{4}+c_{5}+\sqrt{2} c_{6}-\sqrt{2} c_{8}-\sqrt{2} c_{9}-\sqrt{2} \sqrt{2} c_{10}
\end{aligned}
$$

$\operatorname{EXP}(\mathrm{P} 16)=\mathrm{c}_{0}-\mathrm{c}_{1}-\sqrt{2} \mathrm{c}_{3}-\mathrm{c}_{4}+\mathrm{c}_{5}+\sqrt{2} \mathrm{c}_{7}-\sqrt{2} \mathrm{c}_{12}+\sqrt{2} \mathrm{c}_{13}+\sqrt{2} \sqrt{2} \mathrm{c}_{15}$

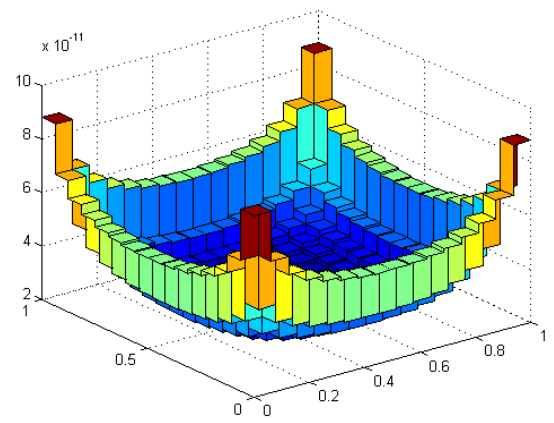

Fig. 6 - Distribuição da densidade superficial de carga em uma chapa plana dividida em 16 segmentos iguais
A Tabela 2 apresenta o valor de alguns coeficientes obtidos através do programa.

\section{TABELA 2 - VALOR DOS COEFICIENTES UTILIZANDO A WAVELET DE HAAR BIDIMENSIONAL}

\begin{tabular}{cc}
\hline Coeficientes & Valor $\times 10^{-10}$ \\
\hline $\mathrm{C}_{0}$ & 0,2903 \\
$\mathrm{C}_{1}$ & $\mathbf{0}$ \\
$\mathrm{C}_{2}$ & 0,0218 \\
$\mathrm{C}_{3}$ & $-0,0218$ \\
$\mathrm{C}_{4}$ & $\mathbf{0}$ \\
$\mathrm{C}_{5}$ & $\mathbf{0}$ \\
$:$ & $:$ \\
$\mathrm{C}_{14}$ & 0,0010 \\
$\mathrm{C}_{15}$ & $-0,0010$ \\
\hline
\end{tabular}

A Fig. 6 apresenta a distribuição de carga em uma chapa quadrada, dividida em 16 segmentos iguais em cada um dos eixos, submetida a um potencial constante de $1 \mathrm{~V}$.

\section{ASPECTOS COMPUTACIONAIS}

A aplicação dos métodos numéricos na engenharia visa facilitar a solução de problemas complexos com um tempo de resposta reduzido. O tempo de início e o término de uma tarefa é então definido (Patterson e Hennessy,2001) pelo tempo de execução. A performance de um determinado programa é definido por:

Performance $=\frac{1}{\text { Tempo_de_Execução }}$

Um bom número de medidas populares, podem ser utilizadas na tentativa de se criar um padrão na medida de performance. O resultado tem sido medições simples, válidas apenas em contextos limitados. Outra alternativa popular para a medida do tempo de execução é o MFLOPS, ou milhões de operações em ponto flutuante por segundo, de acordo com :

MFLOPS $=\frac{\text { Número } \_ \text {de } \_ \text {operações } \_ \text {em } \_ \text {ponto } \_ \text {flutuante }}{\text { Tempo } \_ \text {de } \_ \text {Execução } \times 10^{6}}$

\subsection{Características do sistema computadorizado}

As medidas de performance dos programas desenvolvidos neste trabalho, foram realizadas em um sistema com as seguintes características : 
a) Processador Pentium III, CPU 800MHz;

b) Sistema Operacional Windows NT versão 4.0;

c) Capacidade de memória de 128 Mbytes;

d) Disco rígido de 6.4 Gbytes;

e) Matlab versão 5.0.

\subsection{Medida de performance do programa}

$\mathrm{Na}$ aplicação em uma placa plana finita, medimos o tempo de execução do programa, variando-se o número de divisões em cada um do eixos, medindo-se tanto a quantidade realizada de operações em ponto flutuante como o tempo de execução.

A Tabela 3 mostra os valores obtidos para o tempo de execução total e a quantidade de operações realizadas em ponto flutuante, utilizando como função de expansão a wavelet de Haar.

\section{TABELA 3 - CÁLCULO DAS OPERAÇÕES EM PONTO FLUTUANTE E DO TEMPO DE EXECUÇÃO EM FUNÇÃO DO NÚMERO DE DIVISÕES DA CHAPA}

\begin{tabular}{ccc}
\hline Divisões em & $\begin{array}{c}\text { Operações em ponto } \\
\text { flutuante } \\
\text { cada eixo }\end{array}$ & $\begin{array}{c}\text { Tempo de execução } \\
\text { (Flops) }\end{array}$ \\
\hline 4 X4 & 29.075 & 0,321 \\
$8 \mathrm{X} 8$ & 1.236 .699 & 7,931 \\
$16 \mathrm{X} 16$ & 70.025 .893 & 451,96 \\
\hline
\end{tabular}

\subsection{Melhoria da performance}

Aproveitando-se do fato de que a matriz de Haar é esparsa, (Morettin,1999), reduzimos o tempo de execução inserindo no programa uma comparação que, quando o valor nulo for detectado, a operação entre as matrizes não é realizada. A Tabela 4 apresenta os resultados comparando-se os valores do tempo de execução e do número de operações em ponto flutuante, com e sem a detecção de valores nulos.
TABELA 4 - QUANTIDADE DE OPERAÇÕES EM PONTO FLUTUANTE E TEMPO DE EXECUÇÃO (s), EM FUNÇÃO DO NÚMERO DE DIVISÕES DA CHAPA COM E SEM A DETEÇÃO DOS VALORES NULOS

\begin{tabular}{|c|c|c|c|c|c|}
\hline \multirow[b]{3}{*}{ Divisões } & \multicolumn{4}{|c|}{ Valores nulos } & \multirow{3}{*}{$\begin{array}{c}\text { Diferença } \\
\%) \\
\begin{array}{c}\text { Tempo de } \\
\text { execução }\end{array} \\
\end{array}$} \\
\hline & \multicolumn{2}{|c|}{ Sem detecção } & \multicolumn{2}{|c|}{ Com detecção } & \\
\hline & $\begin{array}{c}\text { Operações } \\
\text { em Flops }\end{array}$ & $\begin{array}{l}\text { Tempo de } \\
\text { execução }\end{array}$ & $\begin{array}{c}\text { Operações } \\
\text { em Flops }\end{array}$ & $\begin{array}{l}\text { Tempo de } \\
\text { execução }\end{array}$ & \\
\hline $4 \mathrm{X} 4$ & 29.075 & 0,321 & 25.491 & 0,250 & 22,12 \\
\hline $8 \times 8$ & 1.236 .699 & 7,931 & 843.483 & 5,488 & 30,80 \\
\hline $16 \times 16$ & 70.025 .893 & 451,96 & 39.748 .261 & 222,60 & 50,75 \\
\hline
\end{tabular}

De acordo com os resultados, reduzimos em média $40 \%$ o tempo de execução do programa.

A Fig. 7 mostra os valores obtidos para o tempo de execução com e sem a detecção de valores nulos, em função do número de divisões da chapa plana finita.

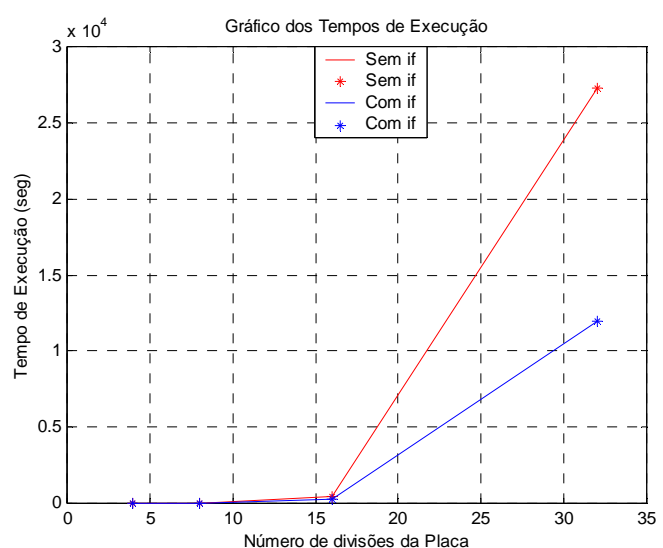

Fig. 7 - Valor do tempo de execução em função do número de divisões com e sem a detecção de valores nulos

Quando a chapa foi dividida em 16 segmentos iguais em cada um dos eixos, foram gerados um total de 256 coeficientes, sendo que $54 \%$ deles são nulos. Aproveitandose do fato que a matriz de Haar é esparsa, aplicando-se a álgebra de matrizes podemos escrever que :

$[\mathrm{H}] \times\left[\mathrm{Z}_{\mathrm{mn}}\right] \times\left[\mathrm{H}^{\mathrm{T}}\right] \times\left[\mathrm{H}^{\mathrm{T}}\right]^{-1} \times[\rho]=[\mathrm{H}] \times[\mathrm{V}]$ 


\subsection{Melhoria da performance com variação de limiar}

A seguir, mostramos a quantidade de elementos não nulos (pontos em cor azul), obtidas para uma chapa plana com 16 divisões em cada um dos eixos através da seleção de um limiar.

As Fig. 8 e 9 apresentam os resultados obtidos variando-se o limiar em $0,01 \%, 0,02 \%$, respectivamente.

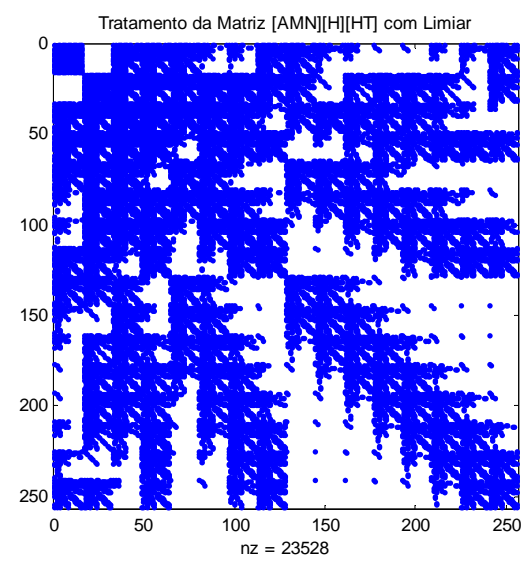

Fig. 8 - Valor do limiar de 0,01\%

(23528 elementos não nulos)

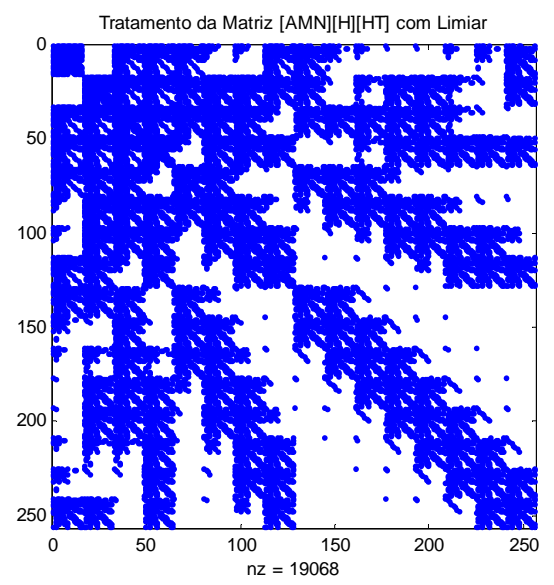

Fig. 9 - Valor do limiar de 0,02\%

(19068 elementos não nulos)

A Tabela 5 apresenta a taxa de variação em porcentagem da quantidade de elementos não nulos, quando aplicamos o limiar selecionado, em relação ao valor inicial de 65.536 pontos.

\section{TABELA 5 - VARIAÇÃO DA QUANTIDADE DE ELEMENTOS EM PORCENTAGEM EM FUNÇÃO DO LIMIAR}

\begin{tabular}{cccc}
\hline \multicolumn{3}{c}{ Elementos } \\
\hline Limiar (\%) & Quantidade & Não Nulos (\%) & Nulos (\%) \\
\hline 0,00001 & 63.088 & 96,26 & 3,74 \\
0,01 & 23.528 & 35,90 & 64,10 \\
0,02 & 19.068 & 29,09 & 70,91 \\
0,05 & 12.232 & 18,66 & 81,34 \\
0,10 & 6.704 & 10,23 & 89,77 \\
\hline
\end{tabular}

A Fig. 10 representa a variação da densidade superficial de carga, para uma chapa plana com 16 divisões em cada um dos lados, em função do limiar selecionado.

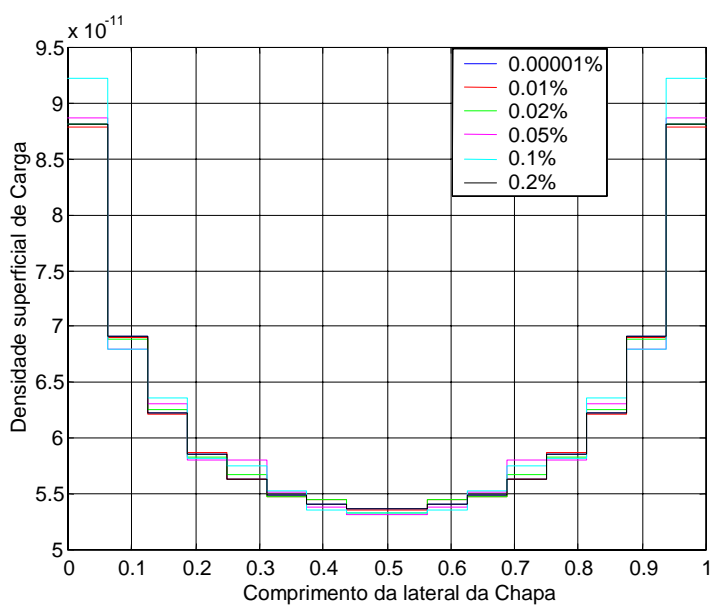

Fig. 10 - Variação da densidade superficial de carga em função do limiar selecionado

A Tabela 6 e a Fig. 11 apresentam a variação do tempo de execução referente à solução do sistema de matrizes, variando-se o número de divisões da chapa, em função do limiar selecionado.

\section{TABELA 6 - TEMPO DE EXECUÇÃO EM FUNÇÃO DO LIMIAR}

\begin{tabular}{cccccc}
\hline \multicolumn{6}{c}{ Tempo de execução (s) } \\
\hline $\begin{array}{c}\text { Divisões } \\
\text { da chapa }\end{array}$ & $0,00001 \%$ & $0,01 \%$ & $0,02 \%$ & $0,05 \%$ & $0,1 \%$ \\
$16 \times 16$ & 0,27 & 0,21 & 0,19 & 0,16 & 0,16 \\
$32 \times 32$ & 25,486 & 11,49 & 8,88 & 4,516 & 2,073 \\
\hline
\end{tabular}




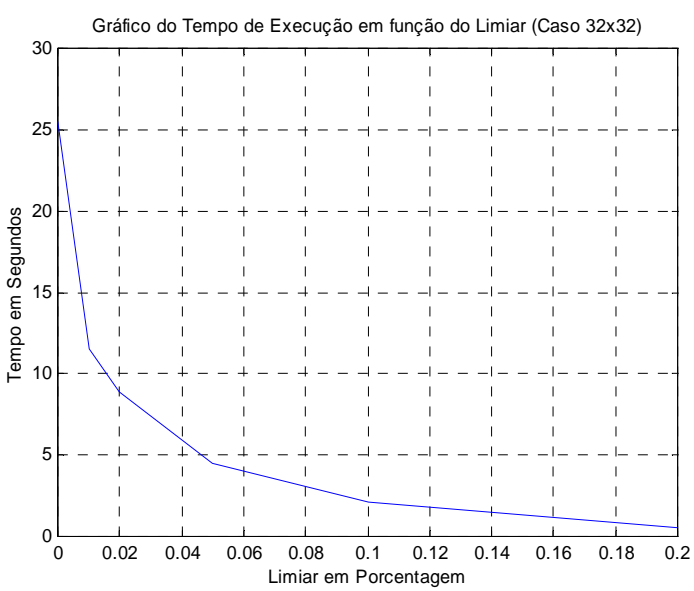

Fig. 11 - Tempo de execução em função do

limiar selecionado (caso 32x32)

Portanto, a variação do limiar permitiu uma redução significativa no tempo de execução, sem alterar significativamente o valor da densidade superficial de carga.

\subsection{Melhoria da performance utilizando a decomposição de Cholesky}

Um método eficiente para o cálculo de matrizes é através da decomposição de Cholesky (Datta,1995), que considera que a matriz é simétrica positiva definida.

Tais matrizes aparecem em uma grande variedade de aplicações, como na solução numérica de problemas de valor inicial pelo método das diferenças finitas, ou pelo método de elementos finitos.

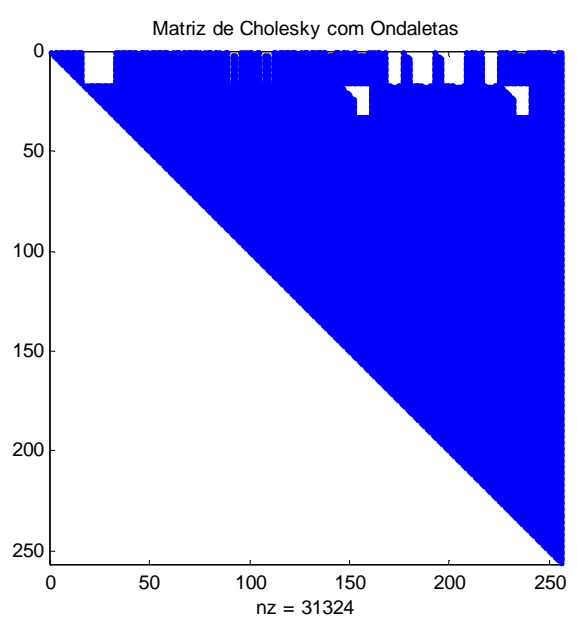

Fig. 12 - Quantidade de elementos após a decomposição de Cholesky $(0,01 \%)$
A decomposição de Cholesky, considera que uma determinada matriz A de ordem NxN, pode ser fatorada em um produto $\mathrm{A}=\mathrm{HH}^{\mathrm{T}}$, sendo que $\mathrm{H}$ é a matriz triangular inferior com elementos diagonais positivos.

A Fig. 12 mostra a quantidade de elementos de forma triangular, obtida após a aplicação de Cholesky com limiar de $0,01 \%$.

Mantendo-se o limiar de $0,01 \%$ (64\% de valores nulos) notamos que após a aplicação da decomposição de Cholesky, o tempo de execução parcial médio para uma chapa plana com 16 divisões em cada eixo, passou de 0,21 para 0,02 (s) e com 32 divisões de 11,49 para 0,351(s), melhorando-se ainda mais o desempenho global do programa.

\section{CONCLUSÃO}

De acordo com os resultados obtidos podemos observar que o método numérico apresentado é extremamente eficiente $\mathrm{e}$ preciso nas soluções de problemas em eletrostática.

Com a metodologia proposta nos dois casos que foram objeto de nosso estudo, considerando-se o produto interno da função de expansão a wavelet de Haar no lugar da função pulso (a função de ponderação Delta de Dirac) e a discretização do domínio da função através de pontos, obtivemos uma variação no cálculo dos coeficientes, que contribuem para o cálculo da densidade superficial de carga, inferior a $0,025 \%$.

Dado o fato de que a matriz das transformadas das wavelets de Haar produz matrizes esparsas, através de alternativas de programação como, por exemplo, a detecção de valores nulos, obtivemos uma diminuição na quantidade de operações em ponto flutuante muito significativa, conseguindo-se uma redução no tempo total de execução do programa de aproximadamente $40 \%$.

Com a seleção de um limiar em porcentagem, que somente é possível de ser implementado quando utilizamos como função de expansão a wavelet de Haar, podemos eliminar alguns coeficientes pela diferença em módulo entre o maior valor positivo e o menor negativo, obtivemos uma melhoria na performance do programa.

No caso da chapa plana quadrada com 16 divisões em cada um dos eixos e um limiar de $0,01 \%$, obtivemos uma redução no tempo de execução de $33 \%$.

Aplicando-se a decomposição de Cholesky, para uma chapa plana $16 \times 16$, limiar $0,01 \%$, o tempo de execução parcial médio das operações entre as matrizes, envolvidas para o cálculo da densidade superficial de carga, reduziu em aproximadamente de 10 vezes. 


\section{REFERÊNCIAS BIBLIOGRÁFICAS}

Aboufadel, E., Schlicker S. (2000). "Discovering Wavelets", 1.ed. New York, John Wiley \& Sons, INC, pp. $23-49$.

Belardi A. A, Cardoso J. R., Sartori C. A. F. (2003). "Application of Haar's Wavelets in the Method of Moment to Solve Electrostatic Problems", Poland, Instytut Maszyn Elektrycznych i Transformatorów, ISEF 2003, pp. $15-20$.

Belardi A. A, Cardoso J. R., Sartori C. A. F. (2003). "Wavelets Application in Electrostatic and their Computing Aspects". Germany, Electric and Magnetic Fields, EMF 2003, pp. 43 - 46.

Chui K. (1992). "A Tutorial in Theory and Applications", 1. Ed., Texas, Academic Press, pp. $206-216$.

Clayton P. R., Syed N. A. (1998). "Introduction to Electromagnetic Fields", 1.ed. New York, McGrawHill, pp. $166-169$.

Constantine A. B. (1989). "Advanced Engineering Eletromagnetics", 2.ed. New York, John Wiley \& Sons, pp. $670-695$.

Datta B. N. (1995). "Numerical Linear Algebra and Applications", 1. ed. New York, Brooks/Cole Publishing Company, pp. $222-225$.

Harrington R. F. (1968). "Field Computation by Moment Methods", 1.ed., New York, Macmillan Company, pp. $1-38$.

Morettin P. A. (1997). "7 Escola de séries Temporais e Econometria”, 1.ed. São Paulo, Edusp, pp. 19 - 38.

Morettin P. A. (1999). “Ondas e Ondaletas” , 1.ed., São Paulo, Edusp, pp. $159-180$.

Newland D. E. (1993). "Random Vibrations, Spectral and Wavelet Analysis",3.ed, Edinburgh, Addison Wesley Longman Limited, pp. 303 - 326.

Patterson D. A., Hennessy J. L. (2001). “Organização e Projeto de Computadores a Interface "Hardware/Software" 1.ed. Rio de Janeiro, Livros Técnicos e Científicos - LTC, pp. $26-45$.

Steinberg B. Z., Leviatan Y. (1995). "On the Use of Wavelet Expansions in the Method of Moments", IEEE Transactions on antennas and propagation, v43, n.8, pp. 802-810. 